\title{
Teoría de juegos aplicada a la competencia innovativa de la industria 4.0
}

\author{
Kevin Alexis Sibrián Sánchez*
}

Sibrián, K. (2020). Teoría de juegos aplicada a la competencia innovativa de la industria 4.0. Revista Activos, 18(2), 91-110. https://doi. org/10.15332/25005278/6261

Recibido: 29 de junio de 2020

Aprobado: 31 de agosto de 2020

\section{Clasificación JEL: C78, M15}

\section{Resumen}

La teoría de juegos, desde su origen hasta la actualidad, es una herramienta utilizada para determinar las múltiples decisiones estratégicas que realizan los jugadores (tomando en consideración que estos son racionales y las reglas del juego se encuentran predefinidas) para alcanzar el máximo beneficio o utilidad, hasta que aquellos se encuentren compitiendo o cooperando y no quieran moverse de esa situación, logrando así el equilibrio de Nash.

* Economista, estudiante de la Maestría en Políticas Públicas de la Universidad de El Salvador, San Salvador.

Correo electrónico: ksibriansanchez@gmail.com 
En ese orden de ideas, ante la importancia de la competencia innovativa en el desarrollo del contexto capitalista a nivel internacional, mediante el auge de la industria 4.0, se justifica la decisión de competir agresivamente por medio de la ciencia y la tecnología para impulsar la aplicación de nuevas innovaciones centradas en la autonomatización de los procesos productivos; además, surge la necesidad de garantizar las condiciones para que estas puedan ser aplicadas efectivamente.

Palabras clave: teoría de juegos, competencia, innovación, imitación e industria 4.0.

\title{
Game theory applied to innovative competition in Industry 4.0
}

\begin{abstract}
Game theory, from its origin to the present day, is a tool used to determine the multiple strategic decisions made by the players (taking into consideration that these are rational and the rules of the game are predefined), to achieve the maximum benefit or utility, until all the players who are competing or cooperating and do not want to move from that situation, achieve the Nash equilibrium. In this order of ideas, highlighting the importance of innovative competition in the development of the capitalist context at international level through the rise of Industry 4.0, the decision to compete aggressively through science and technology to promote the application of new innovations focused on the autonomation of production processes is justified. Additionally, there is a need to ensure the conditions for these to be applied effectively.
\end{abstract}

Keywords: game theory of, competition, innovation, imitation and Industry 4.0. 


\section{Introducción}

La toma de decisiones basada en la teoría de juegos es un área de la microeconomía muy poco explorada, en especial a partir de la competencia innovativa que se desarrolla en la industria 4.0. En ese orden de ideas, esta investigación tiene por objetivo efectuar una profundización teórica a partir del modelo de equilibrio de Nash. Para esto, se analiza la interdependencia estratégica en industrias donde el componente innovativo es un factor clave de la competencia, ya que esto permite a la empresa innovadora, en un instante $t$, obtener una mayor cuota de mercado que sus competidores y un mayor beneficio económico, a partir de la satisfacción de la demanda o por la obtención de una patente.

Por lo anterior, en esta investigación se realiza un abordaje cualitativo a partir de la relación entre competencia, interdependencia estratégica e innovación desarrollada en el actual contexto de la industria 4.0. En este sentido, la investigación está estructurada en tres partes:

1. Un recuento del marco teórico utilizado y los principales representantes en la teoría de juegos (John Nash), innovación (Joseph Schumpeter) e industria 4.0 (Klaus Schwab).

2. Una exposición sobre la aplicación de la teoría de juegos en la toma de decisiones para la negociación tecnológica. Esto se realiza mediante dos modelos: (a) para un instante $t$ en el que dos jugadores deciden si deben innovar o no en la industria de videojuegos; y (b), para una situación en la que un jugador decide innovar antes que otro para la industria farmacéutica.

3. Un análisis sobre el aprovechamiento de las innovaciones generadas en la industria 4.0 para la obtención de mayores niveles de rentabilidad económica. Este análisis parte de la competencia innovativa basada en la teoría de juegos. Además, se destacan las políticas públicas y su papel al garantizar las condiciones para que las innovaciones sean aplicadas efectivamente en un país. 
Finalmente, se concluye sobre los elementos más importantes de la investigación y la necesidad de transformar los modelos estáticos en dinámicos, debido al constante cambio de la sociedad y la tecnología dentro del ámbito económico.

\section{Metodología}

Esta investigación permite determinar si la teoría de juegos es una herramienta analítica adecuada para la toma de decisiones centradas en la competencia innovativa. En este contexto, se relaciona, mediante juegos de interdependencia estratégica, la toma de decisiones para determinar si cooperar o competir, innovar o imitar con el fin de generar ventajas innovativas en una empresa.

Por consiguiente, el método utilizado es el deductivo, el cual parte de una revisión documental sobre investigaciones previas de la teoría de juegos y su aplicación en la realidad para la toma de decisiones centradas en innovación. Así, con base en las variables relacionadas, se formulan conclusiones específicas sobre la aplicación de la teoría de juegos en el actual desarrollo de la industria 4.0.

\section{Marco teórico}

\section{Teoría de juegos}

La teoría de juegos, creada por Neumann y Morgenstern (1944), se centra en la búsqueda de estrategias óptimas que los jugadores (agentes) utilizan para maximizar su utilidad bajo planteamientos cooperativos y no cooperativos. Esta nueva herramienta de análisis para la toma de decisiones estratégicas tuvo una gran influencia en el campo de la economía y la matemática, en especial a partir de la tesis doctoral de Nash (1950), quien, basándose en los trabajos de Neumann, Morgenstern y Kuhn, demostró que existe una solución para juegos estratégicos no cooperativos, donde los jugadores 
obtienen el mejor resultado posible al comprender que se encuentran en una situación de interdependencia estratégica y, por lo tanto, ningún jugador tiene el incentivo de alejarse de aquella, denominada el equilibrio de Nash.

De acuerdo con Álvarez (2015), la teoría de juegos tiene una gran utilidad para analizar el funcionamiento de las estructuras básicas de mercado con un número reducido de oferentes y cuyos resultados dependan de los resultados que los otros obtengan, permitiendo predecir el comportamiento de los jugadores bajo unas determinadas reglas del juego previamente definidas.

A partir de lo anterior, los jugadores forman sus estrategias óptimas y estas pueden cambiar, de acuerdo con Fernández y Parra (2012), según del tamaño de la empresa, el rubro de actividad económica, el mercado, el tipo de juego que realizan el resto de los jugadores, etc. Esta situación conduce a un juego fuertemente competitivo (incluso vengativo) o a uno de alianzas y cooperación que permite a todos los jugadores obtener una equitativa distribución de los beneficios. Por lo tanto, la teoría de juegos tiene un fuerte vínculo analítico con la decisión de competir por parte de las empresas: este es un elemento relevante en la presente investigación.

\section{Competencia innovativa}

El análisis en torno a la competencia y su importancia en el ámbito económico, social y político está frecuentemente atravesado por el debate, debido al impacto que aquella tiene sobre el bienestar social y que, ante la existencia de fallas en el mercado, puede generar la pérdida de una parte de ese bienestar. La competencia surge en un mercado a partir de la interacción continua entre un determinado número de compradores y vendedores que, en su deseo de maximizar sus utilidades y beneficios, buscan tomar las mejores decisiones sujetas a las condiciones con las que cuenta el mercado.

Si el análisis de la competencia se traslada únicamente a los vendedores, estos van a buscar una posición ventajosa sobre el resto de los competidores 
para alcanzar su deseo de maximización de beneficios. La comprensión del comportamiento racional que tienen los vendedores frente a la competencia (elemento fundamental en la teoría de juegos) conlleva a enfocarse en cuatro tipos de ventajas que pueden obtener:

- Ventaja absoluta: este término fue desarrollado por Smith (1776) para indicar la ventaja que tiene un país o empresa al producir una unidad de un bien con menos trabajo que otro país o empresa.

- Ventaja comparativa: basado en la idea de Smith, (Ricardo, 1817) designa la ventaja de un país o empresa en la producción de un bien cuando el coste de oportunidad en la producción de dicho bien en términos de otros bienes es menor en este país o empresas que en otros países o empresas.

- Ventaja competitiva: ampliando mucho más el enfoque de sus predecesores, Porter (1990) establece que una empresa será más eficiente (productiva) en un mercado o industria a medida que la innovación se vuelve el motor de la competitividad en las empresas, incluyendo nuevas tecnologías y formas de hacer las cosas.

- Ventaja innovativa: es un término reciente que surge con la industria 4.0 y, de acuerdo con Basco, Beliz, Coatz y Garnero (2018), se considera como la capacidad que tiene una empresa para aprovechar los conocimientos y tecnologías aplicadas a los procesos productivos para fortalecer las condiciones de las ventajas comparativas y competitivas.

Debido a que el análisis de las ventajas innovativas busca aprovechar las nuevas tecnologías desarrolladas en la industria 4.0, en este caso se aplicará la teoría de juegos con el objetivo de obtener mayores ventajas innovativas (competencia innovativa) para maximizar los beneficios esperados mediante la satisfacción de las demandas exigentes. 


\section{Innovación e industria 4.0}

El concepto de innovación en la literatura económica ha sido ampliamente trabajado, siendo Joseph Schumpeter uno de sus principales exponentes. Este autor subraya la importancia que juega la innovación en la determinación de la prosperidad de la sociedad y explica la dinámica cambiante del proceso de innovación (introduciendo el concepto de "destrucción creadora") como producto del proceso evolutivo del capitalismo y del papel que juega el empresario dentro del mismo.

A partir de lo anterior, se plantean las siguientes categorías de innovación: la introducción de un nuevo bien o una nueva calidad de un bien; la utilización de un nuevo método de producción; la apertura de un nuevo mercado; la conquista de una nueva fuente de aprovisionamiento de materias primas o bienes semimanufacturados; la creación de una organización para cualquier industria; $y$, las innovaciones de mercadotecnia que implican la puesta en práctica de nuevo métodos de comercialización (Schumpeter, 2015; OCDE, 2006). Tomando en cuenta la importancia de la innovación en la dinámica capitalista Schumpeter (2015) explica que la única competencia que tiene valor es la que implica la aparición de algunas de las innovaciones enumeradas, es decir, la competencia más importante es la que da lugar a una superioridad en el costo o en calidad. Es aquí donde la innovación adquiere importancia como motor de la industria 4.0 y de la toma de decisiones para las negociaciones tecnológicas.

Asimismo, esta cuarta revolución industrial consiste en la habilidad de internet para controlar el mundo físico mediante la sincronización de equipos, procesos y personas en la industria, modificando el modelo tradicional de manufactura que posibilita el surgimiento de nuevos mercados de productos y servicios. Además, la industria 4.0 se encuentra caracterizada porque las empresas deciden autonomatizar sus procesos productivos mediante la aplicación de diversas tecnologías como macrodatos, computación en la nube, robotización, internet de las cosas, impresión 3D, entre otras. 
De acuerdo con todo lo expuesto, la decisión de invertir estratégicamente en innovación se encuentra fuertemente ligada al deseo de obtener mayores ventajas en el contexto de la actual industria 4.0. Si bien se ha realizado un abordaje sobre la importancia que tiene la decisión de innovar (siendo más que un deseo, una necesidad), es necesario abordarla a partir de un modelaje de la teoría de juegos para determinar la estrategia óptima de los jugadores en las negociaciones tecnológicas.

\section{Modelos de teoría de juegos aplicados a las negociaciones tecnológicas}

\section{Negociaciones tecnológicas para un instante $t$}

En el contexto actual donde la globalización se extiende a múltiples aspectos de la vida humana, sin excluir el ámbito económico, la teoría de juegos provee un marco de análisis para las negociaciones tecnológicas (específicamente, aquellas que realizan actividades de I+D) a partir de la formalización de las opciones que un jugador tiene a su disposición para maximizar la utilidad, tomando en consideración las decisiones de los otros jugadores y el dilema de cooperar o no cooperar con sus rivales. Según Fernández y Parra (2012), es en este escenario donde, a partir de la intensificación del proceso de globalización, aparece la teoría de juegos de Nash con el fin de recrear los componentes esenciales en un proceso de negociación tecnológica de cara a un entorno fuertemente competitivo.

Para encontrar la solución óptima al dilema de innovar o no innovar, se hará uso de un juego (figura 1) que consta de los siguientes supuestos:

- Existen dos jugadores, es decir, dos empresas que deciden entre innovar o no innovar.

- Los dos jugadores deben decidir, en un instante $t$, entre innovar o no innovar, por lo tanto, ningún jugador conoce los movimientos del otro, existe información imperfecta. 
- Las reglas del juego se encuentran previamente definidas e invariantes, además los jugadores las conocen, es decir que existe información completa.

- Se asume que los jugadores son racionales.

Figura 1. Dilema de innovación en la industria de videojuegos

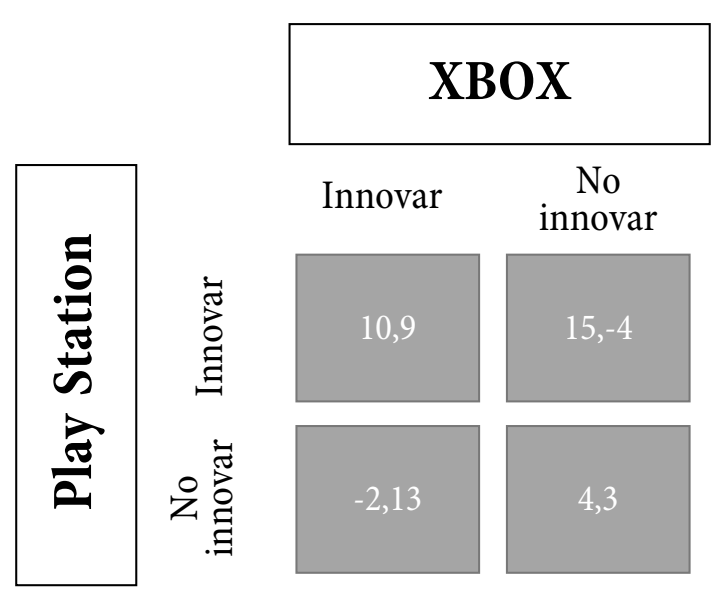

Fuente: elaboración propia.

Tomando en consideración los supuestos, Play Station y Xbox deben de decidir si introducen innovaciones o no en sus nuevas consolas, juegos, complementos, etc. En un primer caso, si las dos empresas deciden optar por un juego cooperativo, en el que ninguna innova, entonces los beneficios serán repartidos "equitativamente" (el juego se resuelve en el tramo 4,3 ), conduciendo a la formación de alianzas y, por ende, a un oligopolio no competitivo. Pero, de acuerdo con Nash (1950), este tipo de solución al dilema de innovación no es óptima, debido a que existe el deseo latente de romper la alianza e introducir innovaciones para obtener una mayor ganancia, corriendo el riesgo de perder más de lo que hubiera perdido en el pacto. Sin embargo, este riesgo no se genera en esta industria debido a los condicionantes de Fernández y Parra (2012) previamente expuestos. Por lo tanto, si Play Station decide romper el acuerdo, introduciendo una 
nueva generación de consolas y videojuegos con realidad ampliada, obtendrá una mayor ganancia esperada debido a que Xbox aún se mantiene cumpliendo el acuerdo (el mismo análisis se puede hacer si Xbox rompe la alianza mientras que Play Station no).

La falla existente en los juegos cooperativos para esta industria radica en que los jugadores no solo compiten por medio de los precios, sino que dicha competencia se ve determinada por el grado de innovación que les garantiza quiénes se mantendrán en el mercado. En el dilema de innovación, si una de las empresas, ya sea Xbox o Play Station, decide acomodarse sin innovar productos y haciendo siempre lo mismo sin tener el riesgo a perder, entonces tenderá inevitablemente a desaparecer, debido a la insatisfacción que recibe el consumidor por el bajo grado de innovación incorporado en sus productos, incluso llevando a que deje de consumirlos.

El equilibrio de Nash deseado en la figura 1 se obtiene cuando Xbox y Play Station deciden innovar (ubicándose en el tramo 10,9), ya que al considerar que su oponente es racional, los jugadores buscarán las mejores estrategias en el mercado, introduciendo innovaciones de forma continua para obtener una mayor porción de los beneficios. De esta forma, en un tipo de juego no cooperativo, se genera un doble beneficio en la industria: (1) el consumidor tendrá a su disposición una gran gama de productos y servicios con diferentes precios, diseños y calidad, incrementando su frontera de elección y la satisfacción obtenida de la competencia; y (2) sus beneficios serán aún mayores que en una situación en que los jugadores decidan formar alianzas para no innovar.

La lógica detrás de lo expuesto, a partir de las funciones de pago ad $h o c$, reside en que la competencia agresiva en innovación es el motor que estimula la oferta y demanda de los productos, garantizando la obtención de ventajas innovativas mayores traducidas en una mayor cuota de mercado y, por ende, en beneficios. En conclusión, el equilibrio de Nash es alcanzado en la figura 1 debido a que ningún jugador dejará de innovar, se encuentra en la mejor situación posible al tomar en cuenta la interdependencia estratégica existente. 


\section{Negociaciones tecnológicas en instantes $t$ diferentes}

Hasta el momento se ha analizado un tipo de juego en el que ambos jugadores deben de decidir, en un instante $t$, si competir o no en innovación, obteniendo la solución óptima para ambos jugadores. Pero, en la realidad, esto no es así, debido a que las decisiones de innovar pueden surgir antes en un determinado jugador que en otro, provocando que, en una industria exista un innovador (pionero en la utilización de un invento en la industria) y un imitador (aplica la innovación producida por empresas de la competencia, por ejemplo, a partir de licencias) (Schumpeter, 2015). Por esta razón, el análisis en la teoría de juegos debe de modificarse, incluyendo los siguientes supuestos:

- Los jugadores utilizan un número finito de estrategias para innovar, las cuales pueden realizarse en instantes diferentes, y estarán enfocadas en innovar o imitar.

- La industria es dinámica, por lo tanto, las empresas están innovando para ofrecer el mejor producto, el más reciente y el que incorpore las últimas innovaciones.

Asumiendo que dos jugadores deciden realizar la introducción de una nueva innovación al mercado, por la cual cada uno obtiene una patente para defender los derechos de propiedad de su acción innovadora, puede haber dos situaciones: (1) si la decisión de innovar se realizó de forma conjunta, no cooperativa, por los dos jugadores, entonces ambas tendrán un producto diferenciado con un determinado nivel de beneficio; y (2) si una de las empresas decide innovar antes que otra, entonces habría que analizar si no existe un incumplimiento de la patente.

De esta forma, Cukierman (1994) explica que la teoría de juegos conduce a un análisis centrado en la respuesta del segundo jugador $(y)$ respecto de la innovación del primero $\left(y^{*}\right)$, en donde el segundo jugador puede decidir: 
- Imitar la innovación: en esta situación el segundo jugador viola la patente del primero $\left(y<y^{*}\right)$, teniendo la obligación de pagar una licencia para poder comercializar su producto.

- Introducir una nueva innovación: las características de la innovación del segundo jugador son tales que refleja una innovación importante $\left(y>y^{*}\right)$, por lo tanto, las dos empresas terminarán formando parte de un nuevo oligopolio.

En este tipo de juegos, lo esencial radica en determinar la política de patentes óptimas, esta debe incentivar al primer jugador a innovar, pero al mismo tiempo no ser tan fuerte como para reducir o eliminar el deseo competitivo del resto de jugadores. Por lo tanto, la política de patentes óptimas debe tomar en cuenta dos elementos:

- Longitud de la patente: según Castillo y Guevara (1999), es el periodo durante el cual se concede la patente al inventor.

- Amplitud de la patente: según Klemperer (1990), es el conjunto de características en el espacio del producto que infringen el producto patentado.

\section{Teoría de juegos y políticas de patentes óptimas}

Betegon (2004) desarrolla un ejemplo de este tipo de juegos para la industria farmacéutica, en el que considera a tres jugadores: (1) empresa farmacéutica; (2) Estado que concede patentes para productos y procedimientos, con capacidad de producción ineficientes o inexistentes; y (3) Estado que concede patentes para productos y procedimientos, con capacidad de producción. A partir de esto, asume que los jugadores son racionales y la información es completa, lo que le permite clasificar el juego como no cooperativo, así lo representa de forma extensiva (figura 2). 
Figura 2. Teoría de juegos aplicada a la industria farmacéutica

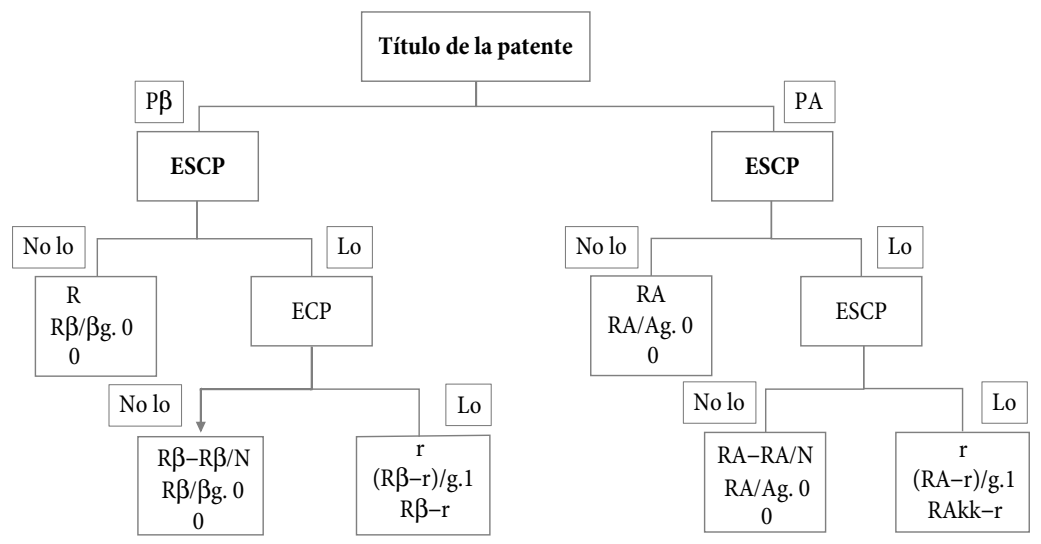

Fuente: Betegon (2004, p. 17).

Donde: $P \beta$ significa precios bajos; $P A$, precios altos; $R \beta$, presupuesto bajo; $R A$, presupuesto alto; $r$, remuneraciones; $L O$ implica que se concede licencia obligatoria; y, no LO significa que no se concede licencia obligatoria para la comercialización del producto patentado. Por último, en la figura 2 se incluyen las siglas ESCP (Estado miembro de la Organización Mundial del Comercio sin capacidad de producir) y esc (Estado miembro de la Organización Mundial del Comercio con capacidad de producir).

De acuerdo con Betegon (2004), los tres jugadores pueden decidir entre las siguientes estrategias:

- Empresa farmacéutica: los pagos que recibe dependen del precio que fije (pueden ser $P A$ o $P \beta$ ), el coste que incurra por alterar el juego a través de presiones a otros jugadores, y las remuneraciones $(r)$ que reciba por el producto al que se le aplique la licencia obligatoria. La decisión de la empresa, teniendo el monopolio sobre el producto patentado, es colocar un precio alto para incentivar el comercio paralelo y el sistema de control de precios de otros países, por lo 
tanto, no hay discriminación de precios entre países desarrollados y en vías de desarrollo.

- ESCP: parte de un presupuesto (puede ser $R A$ o $R \beta$ ) determinado por factores como recursos económicos, la incidencia que tiene el medicamento sobre la enfermedad y el coste de oportunidad, es decir, la rentabilidad de unos tratamientos frente a otros. Tomando en consideración estas variables, el ESCP decide si conceder o no la licencia obligatoria, según las condiciones siguientes:

$\triangleright$ En caso de $L O$, podrá comprar cierta cantidad de medicamentos, pero tomará cierto tiempo para tenerlos a su disposición.

$\triangleright$ En caso de no $L O$, podrá comprar los medicamentos de acuerdo con el presupuesto que destine y el precio que fije la farmacéutica, teniendo los medicamentos de manera inmediata ya que se lo compra al dueño de la patente.

Por lo tanto, el EsCP debe de valorar entre el precio que cobra la farmacéutica (siendo $P A$ ) y el productor genérico, y qué tanto se tarda en tener a su disposición los medicamentos.

- ECP: si el ESCP decide aprobar la $L O$, de igual forma, debe decidir si concederá licencias o no. Para esto tendrá en cuenta: (1) los beneficios directos producto de la venta de los medicamentos; y (2) los beneficios indirectos, obtenidos por el acceso a la tecnología y la creación de los puestos de trabajo, implicados en la producción de medicamentos. También se evaluará la presión que el titular de la patente pueda ejercer.

El ingreso que obtenga dependerá del presupuesto que el ESCP destine a la compra de medicamentos genéricos, menos lo que el ECP remunere $(r)$ a la farmacéutica por el uso de la patente. Este ingreso será bajo $(R \beta-r) \mathrm{o}$ alto $(R A-r)$, según los precios que fije la farmacéutica. Sin embargo, serán los precios altos los que incentiven a usar la licencia. Para determinar el 
número de unidades vendidas, se dividirá el ingreso entre el precio de venta del ECP $(g)$, donde $g<\beta g<A g$.

El marco analítico y de modelación de la teoría de juegos en las negociaciones tecnológicas, a partir de las funciones de pago ad hoc, para diferentes industrias es muy amplio, ya que define las reglas del juego con las que los jugadores se enfrentan en un mercado de interdependencia estratégica sumamente competitivo y permite la obtención del equilibrio de Nash que maximiza la utilidad de todos los jugadores. Además, advierte del papel fundamental que juega la innovación, ampliado y acelerado exponencialmente por el proceso de globalización, y sugiere que las empresas deben de seguir invirtiendo de forma permanente, porque de lo contrario no podrán obtener ventaja innovativa alguna y serán absorbidos por la competencia.

\section{Competencia innovativa en el contexto de la industria 4.0}

A partir de la teoría de juegos se ha resaltado que la innovación y la competencia son elementos fundamentales para la maximización de los beneficios en las empresas, en especial con el desarrollo actual de la industria 4.0 caracterizada por la formación de una industria inteligente que autonomatiza los procesos productivos, una industria predictiva de monitoreo a distancia, la aplicación de realidad ampliada y la introducción continua de nuevas innovaciones para garantizar la calidad de los procesos y productos. De esta manera, las empresas buscan la construcción de ventajas innovativas, pero requieren de un nuevo contrato social tecnológico, capaz de conjugar los esfuerzos de Estados inteligentes, actores privados dinámicos y una comunidad laboral y científica que articule más plenamente sus tareas para el fomento de la innovación estratégica (Basco, Beliz, Coatz y Garnero, 2018).

En ese orden de ideas, la industria 4.0 necesita que las reglas del juego dejen de ser estáticas, ya que se encuentra en un contexto de cambios 
generados por las innovaciones, las necesidades de la industria y el marco institucional. Lo anterior implica que, en un momento $t_{0}$, todos los jugadores pueden tener conocimiento de las reglas del juego (existe infomación completa), pero a medida que estos toman diferentes decisiones que maximicen su situación inicial de interdependencia estratégica, las condiciones a las que se enfrentan pueden ir cambiando y, por ende, sus funciones de pago para un instante $t_{n}$, debido a cambios en la "naturaleza" y el "azar", incluso generando que exista información incompleta.

A partir del auge de la industria 4.0 y la fuerte intensidad con que la destrucción creadora se aplica en las industrias tecnológicas, los modelos estáticos se han desplazado o actualizado a un enfoque dinámico, donde el componente tecnológico y el desarrollo de la ciencia son factores fundamentales de la competencia e impulsan el comportamiento de la actividad capitalista. Esto es evidente en la competencia ejercida en el escenario mundial, donde las naciones han tomado el liderazgo de la industria 4.0 al dar prioridad a la ciencia y tecnología como motores del desarrollo, por ejemplo:

- Digitalización: países como Singapur, Finlandia, Suecia, Noruega y Estados Unidos han aprovechado la conectividad, el capital humano, el uso del internet de las cosas, la integración de la tecnología digital y los servicios públicos digitales para impulsar las ventajas innovativas en la digitalización del sector manufacturero (Foro Económico Mundial, 2016).

- Robotización: la inclusión de robots en la cadena de producción y distribución ha permitido autonomatizar tareas que antes eran circunscriptas al domino humano y a máquinas (controladas o supervisadas por humanos). De acuerdo con la Federación Internacional de Robótica (2020), los países con el puntaje más alto en cuanto a densidad de robots utilizados en la industria son Singapur, Corea del Sur, Japón y Alemania. 
Es tal la importancia de la innovación en las decisiones que, en América Latina, existen seis empresas unicornios (Mercado Libre, PagSeguro, B2W Digital, Nubank y Rappi) muy exitosas en la aplicación de las nuevas tecnologías, garantizando una mayor capacidad para adaptarse proactivamente a las necesidades del mercado (Mafra, 2020). Estas empresas innovan consecutivamente en sus productos y servicios para entregar la mayor calidad y una relación más directa al cliente, de tal forma que logran posicionarse de forma óptima en el mercado.

Al mismo tiempo, surge la necesidad de proveer las condiciones para que las empresas puedan introducirse de forma efectiva a la actual industria 4.0, así las reglas del juego deben de cambiar a tal punto que los jugadores adecuen sus decisiones de innovación a la competencia generada en el mercado internacional. Por esta razón, la política pública es una de las herramientas necesarias para impulsar dichas condiciones de inclusión a la industria 4.0. Entre algunos ejemplos se encuentran:

- Políticas de educación que garanticen el aprendizaje de las habilidades digitales necesarias en la industria 4.0, permitiendo la generación de profesionales de calidad y con formación avanzada en el actual contexto de innovación.

- Políticas para formación de clústeres tecnológicos que fomenten la obtención de ventajas competitivas y, al mismo tiempo, permitan vincular la cadena de valor y la transferencia de información y tecnologías de forma más rápida (Porter, 1990); así como la obtención de ventajas innovativas que garanticen la ciber-seguridad y la logística 4.0 (Basco et al., 2018).

- Políticas para la aplicación de recursos del futuro centradas en fomentar el uso eficiente de los recursos con bajas emisiones de carbono para gestionar vías de desarrollo deseables a largo plazo. 


\section{Conclusiones}

Hasta el momento se ha demostrado la amplitud, generalidad y suficiencia teórica que brinda la teoría de juegos para diferentes situaciones en un ámbito multidisciplinario. Esta capacidad analítica que otorga es indispensable para la formación de economistas, financieros, políticos, administradores, sociólogos, psicólogos, etc., puesto que permite la observación, el estudio y la formalización de las opciones del agente económico ante una situación de interdependencia estratégica y que, bajo ciertos supuestos, puede llevar a enfrentar una multiplicidad de respuestas posibles, en la que solo una respuesta consigue el equilibrio de Nash.

No obstante, la teoría de juegos tiene ciertas limitaciones, por ejemplo:

- Asume que las reglas del juego se encuentran predefinidas y permanecen invariantes. Esto conlleva a una sobre simplificación en la toma de decisiones de los agentes, debido a que el marco en que se enfrentan los jugadores en el mercado internacional, y sobre todo en la industria 4.0 actual, se encuentra en constante cambio, generando que las funciones de beneficio esperadas (funciones de pago) cambien continuamente, así como las decisiones de los jugadores. A causa de esto, se debe de prestar una mayor atención al conjunto de elementos que llevan a que los agentes se enfrenten o cooperen en un mercado.

- La dificultad que presenta el análisis del comportamiento de los agentes, sus decisiones futuras y los ejes de sus intereses se vuelve cada vez mayor. Esto se debe a que el deseo de los jugadores de maximizar la utilidad o el beneficio no solo depende de factores económicos (traducido en una mayor riqueza), también de factores sociales, psicológicos, políticos, tecnológicos, culturales, etc., como la afiliación, el reconocimiento, la autorrealización y el conocimiento.

- La racionalidad de los jugadores es un supuesto que adquiere mayor complejidad, debido a que en la realidad los agentes económicos no 
cuentan con información perfecta de los jugadores ni tampoco con información completa sobre las reglas del juego. Al mismo tiempo, pese a la crítica de Lucas (1981), citado en Contreras (1995), sobre las expectativas racionales, es improbable asumir que un jugador es igual o al menos tan racional a otro.

En síntesis, aunque la teoría de juegos presenta estas limitantes, aún es una herramienta capaz de obtener información detallada, a partir de su abstracción, sobre las decisiones de negociación tecnológica que toman los agentes económicos. Todo esto ocurre en un contexto en el que se intensifican los procesos de globalización, la apertura económica y el desarrollo de la industria 4.0; por lo que, dichos agentes buscan alternativas para sobrevivir en un mercado caracterizado por la competencia innovativa, continua y agresiva.

\section{Referencias}

Álvarez, P. (2015). Teoría de Juegos. Cantabria: Universidad de Cantabria.

Basco, A., Beliz, G., Coatz, D. y Garnero, P. (2018). Industria 4.0: Fabricando el Futuro. Banco Interamericano de Desarrollo.

Betegon, L. (2004). Las licencias obligatorias en materia farmacéutica en la Organización Mundial del Comercio: un análisis desde la teoría de juegos. Universidad de Salamanca, Departamento de Economía Aplicada 2.

Cantillo, M., y Guevara, P. (junio de 1999). Longitud y Amplitud óptima de patentes: un mecanismo para incentivar la innovación. https://www.incae.edu/ES/clacds/ publicaciones/pdf/cen1803.pdf

Contreras, H. (1995). Robert Lucas, las expectativas racionales y la macroeconomía. Economía Informa, (243), 38-40. https://biblat.unam.mx/es/revista/ economia-informa/articulo/robert-lucas-las-expectativas-racionales-y-lamacroeconomia

Cukierman, P. (1994). Los determinantes de la competitividad de la Industria Química de Base en España. Departamento de Economía Aplicada de la Universitat Autònoma de Barcelona. 
Federación Internacional de Robótica [International Federation of Robotics]. (2020). World Robotics Report 2020. https://ifr.org/ifr-press-releases/news/record-2.7million-robots-work-in-factories-around-the-globe\#: :text=Sales $\% 20$ of $\% 20$ new\%20robots\%20remain,highest\%20sales\%20volume\%20ever\%20recorded.

Fernández, J., y Parra, M. (18 de marzo de 2012). La teoría de juegos de Nash en los modelos de negociación tecnológica. Ingeniría Solidaria, 8(14), 89-93.

Foro Económico Mundial. (2016). Informe Global de Tecnología de la Información 2016. Foro Económico Mundial.

Klemperer, P. (1990). How broad should the scope of patent protection be? RAND Journal of Economics, 21(1), 113-130. https://doi.org/10.2307/2555498

Mafra, É. (23 de febrero de 2020). ¿Qué son las empresas unicornio? https://rockcontent.com/es/blog/empresas-unicornio/

Nash, J. (1950). Non-Cooperative Games. Annals of Mathematics.

Neumann, J., y Morgenstern, O. (1944). Theory of Games and Economic Behavior. Princeton University Press.

Organización para la Cooperación y el Desarrollo Económico [OCDE]. (2006). Manual de Oslo: Guía para la recogida e interpretación de datos sobre innovación (3. ${ }^{\text {a }}$ ed.). Grupo Tragsa.

Porter, M. (1990). The competitive advantage of nations. Harvard Business Review. Ricardo, D. (1817). The Principles of Political Economy and Taxation. John Murray. Schumpeter, J. (2015). Capitalismo, Socialismo y Democracia. Barcelona, Página Indómita.

Schwab, K. (2016). La cuarta revolución industrial. Debate.

Smith, A. (1776). An Inquiry into the Nature and Causes of The Wealth of Nations. Harriman Houses. 